\title{
Predictive Value of Calvarial Fracture for Delayed Epidural Hematoma following Decompressive Craniectomy
}

W e read with great interest the article by Talbott et $\mathrm{al}^{1}$ describing the predictive value of patterns of skull fractures on preoperative CT for delayed epidural hematoma. Although we agree with the principal findings of the study, we would like to clarify some important clinical points to further increase the practical value of the article. First, the authors stated that all patients developing a delayed epidural hematoma had a contralateral calvarial fracture on preoperative CT at the site where the delayed epidural hematoma subsequently formed. It is incontrovertible that almost all traumatic epidural hematomas are accompanied by skull fractures; however, there are still some patients with traumatic epidural hematoma without skull fracture or in whom the fracture cannot be detected by CT. ${ }^{2}$ Therefore, we think that the contralateral calvarial fracture could not have perfect sensitivity (100\%) for subsequent development of delayed epidural hematoma as stated by the authors.

Second, epidural hematomas mainly occur due to injuries of blood vessels under the bone, so calvarial fractures near blood vessels are apt to cause epidural hematoma. Therefore, we think that the location of the fracture might be more important than fractures involving 2 or more bone plates, an idea strongly supported by the authors. Last, although the sensitivity was $100 \%$ according to the article, only 12 of all 55 patients with contralateral calvarial fracture developed delayed epidural hematoma. We

http://dx.doi.org/10.3174/ajnr.A4216 admit that the presence of a calvarial fracture contralateral to the anticipated side of craniectomy has a higher risk of delayed epidural hematoma, but it may not be the only clue. Are there some other factors that could help make a more accurate judgment? More large and well-conducted clinical trials may be required to address this issue.

\section{REFERENCES}

1. Talbott JF, Gean A, Yuh EL, et al. Calvarial fracture patterns on CT imaging predict risk of a delayed epidural hematoma following decompressive craniectomy for traumatic brain injury. AJNR Am J Neuroradiol 2014;35:1930-35

2. Su TM, Lee TH, Chen WF, et al. Contralateral acute epidural hematoma after decompressive surgery of acute subdural hematoma: clinical features and outcome. J Trauma 2008;65:1298-302

Q.-p. Wang Department of Neurosurgery West China Hospital Sichuan University Chengdu, China Department of Neurosurgery Dujiangyan People's Hospital Dujiangyan Medical Center Dujiangyan, China

C. You

Department of Neurosurgery West China Hospital Sichuan University Chengdu, China 